\title{
Avaliação e manejo da dor oncológica crônica em unidade de internação pediátrica*
}

Assessment and management of chronic oncologic pain in a pediatric inpatient unit

Evaluación y manejo del dolor oncológico crónico en una unidad de internación pediátrica

\section{Thiago Privado da Silva ${ }^{\mathrm{I}}$, Laura Johanson da Silva ${ }^{\mathrm{II}}$, Ítalo Rodolfo Silva ${ }^{\mathrm{III}}$ Maria José Carvalho Ferreira ${ }^{\mathrm{IV}}$, Luana dos Santos Costa ${ }^{\mathrm{v}}$, Joséte Luzia Leite (In Memorian) ${ }^{\mathrm{NI}}$}

\begin{abstract}
Resumo: Objetivo: compreender a complexidade da avaliação e do manejo da dor oncológica crônica da criança hospitalizada. Método: estudo qualitativo baseado na Teoria da Complexidade e na Grounded Theory. Participaram da pesquisa 21 profissionais de saúde. A entrevista semiestruturada e a observação não participante foram usadas para coletar os dados. A análise seguiu as etapas de codificação. Resultado: a avaliação da dor pelos profissionais está embasada no relato do familiar, da criança e na observação do comportamento da mesma. Utilizaram-se escala analógica de cores, escala de faces e escala numérica. A analgesia farmacológica é a prescrição médica e na não farmacológica realizam-se lúdico, conversa, massagem, banho, compressa morna ou fria e promoção de conforto. Conclusão: a avaliação e o manejo da dor oncológica crônica da criança hospitalizada solicitam dos profissionais de saúde preparo técnico e científico para lidar com aspectos objetivos e subjetivos que envolvem esse cuidado.

Descritores: Enfermagem Pediátrica; Dor Crônica; Medição da Dor; Manejo da Dor; Neoplasias
\end{abstract}

Abstract: Objective: to understand the complexity of the assessment and management of chronic oncologic pain in hospitalized children. Method: qualitative study based on Complexity Theory and Grounded Theory. A total of

\footnotetext{
${ }^{\text {I }}$ Enfermeiro. Doutor em Enfermagem. Universidade Federal do Rio de Janeiro, Macaé, Rio de Janeiro, Brasil. E-mail: thiagopsilva87@gmail.com Orcid: http://orcid.org/0000-0002-7744-8319

II Enfermeira. Doutora em Enfermagem. Universidade Federal do Estado do Rio de Janeiro, Rio de Janeiro, Rio de Janeiro, Brasil. E-mail: lauraenfaunirio@gmail.com Orcid: http://orcid.org/0000-0002-4439-9346

III Enfermeiro. Doutor em Enfermagem. Universidade Federal do Rio de Janeiro, Macaé, Rio de Janeiro, Brasil. E-mail: enf.italo@gmail.com Orcid: http://orcid.org/0000-0002-2882-1877

IV Enfermeira. Mestre em Enfermagem. Universidade Federal do Rio de Janeiro, Rio de Janeiro, Rio de Janeiro, Brasil. E-mail: maria.jcarvalho@live.com Orcid: http://orcid.org/0000-0002-6165-8709

V Enfermeira. Mestre em Enfermagem. Universidade Federal do Rio de Janeiro, Rio de Janeiro, Rio de Janeiro, Brasil. E-mail: luanaufrj@gmail.com Orcid: http://orcid.org/0000-0001-7314-3676

VI Enfermeira. Doutora em Enfermagem. Universidade Federal do Rio de Janeiro, Rio de Janeiro, Rio de Janeiro, Brasil. E-mail: joluzia@gmail.com Orcid: http://orcid.org/0000-0002-9302-0240
}

* Extraído da tese "Gerenciamento do cuidado de enfermagem à criança hospitalizada com dor oncológica crônica", Programa de PósGraduação em Enfermagem da Escola de Enfermagem Anna Nery, Universidade Federal do Rio de Janeiro, 2016. 
21 health professionals participated in the research. Semi-structured interview and non-participant observation were used to collect the data. The analysis followed the coding steps. Result: the pain assessment performed by professionals is based on the relative's report, the child's report and the observation of the child's behavior. Analog color scale, face scale and numerical scale were used. Pharmacological analgesia is the medical prescription; and, in non-pharmacological, ludic aspects, conversation, massage, bathing, warm or cold compress and comfort promotion are performed. Conclusion: the assessment and management process of chronic oncologic pain in hospitalized children requires that health professionals have technical and scientific preparation to deal with objective and subjective aspects that involve this care.

Descriptors: Pediatric Nursing; Chronic Pain; Pain Measurement; Pain Management; Neoplasms

Resumen: Objetivo: comprender la complejidad de la evaluación y el manejo del dolor oncológico crónico en niños hospitalizados. Método: estudio cualitativo basado en la Teoría de la Complejidad y la Teoría Fundamentada. La investigación abarcó 21 profesionales de la salud. Para recoger los datos, se utilizó la entrevista semiestructurada y la observación no participante. El análisis siguió los pasos de codificación. Resultado: la valoración del dolor por parte de los profesionales se basa en el informe del pariente y del niño, así como la observación del comportamiento del niño. Se utilizaron escala de color analógica, escala facial y escala numérica. La analgesia farmacológica es la prescripción médica; y, en la no farmacológica, se realizan ludicidad, conversación, masajes, baños, compresas calientes o frías y promoción del confort. Conclusión: la evaluación y el manejo del dolor oncológico crónico en niños hospitalizados requiere que los profesionales de la salud tengan una preparación técnica y científica para abordar los aspectos objetivos y subjetivos que involucran este cuidado.

Descriptores: Enfermería Pediátrica; Dolor Crónico; Dimensión del Dolor; Manejo del Dolor; Neoplasias

\section{Introdução}

No Brasil, o câncer em crianças e adolescentes (de 0 até 19 anos) é considerado raro quando comparado com os tumores do adulto, pois corresponde entre $2 \%$ e $3 \%$ de todos os tumores malignos, destacando-se como a mais importante causa de óbito nos países em desenvolvimento. ${ }^{1} \mathrm{Na}$ oncologia pediátrica, a literatura destaca a dor como um dos sintomas mais prevalente e angustiante vivenciado pela criança. ${ }^{2-4}$ Sua ocorrência pode estar relacionada ao próprio câncer e/ou aos procedimentos diagnósticos e terapêuticos aos quais a criança é submetida durante o tratamento. ${ }^{5}$ Estudo revelou que $52 \%$ das crianças com leucemia apresentaram dor crônica ao longo do seu tratamento e os locais mais comuns de dor foram costas $(8 \%)$, pernas $(8 \%)$, estômago $(8 \%)$, peito $(6 \%)$, braços $(4 \%)$ e cabeça $(4 \%){ }^{3}$

A literatura ressalta que a presença da dor crônica em crianças com doença crônica, por exemplo, o câncer, pode afetar negativamente vários aspectos da vida infantil, incluindo a 
prática de atividades físicas, frequência escolar, padrão de sono, interação familiar, humor, entre outros aspectos..$^{5}$ A esse respeito, o estudo supracitado identificou que as crianças que manifestaram dor oncológica crônica apresentaram dificuldades no âmbito extracurricular (16,7\%), doméstico (14,6\%), social $(12,6 \%)$, sono $(12,6 \%)$ e acadêmico $(12,5 \%){ }^{3}$

Portanto, é importante que a avaliação e o manejo da dor oncológica crônica sejam realizados com base em um pensamento que valorize a natureza multidimensional da dor, a complexidade das relações humanas e o trabalho em equipe. Nesse contexto, a Teoria da Complexidade surge como possibilidade para compreender o desafio que envolve o cuidado à criança hospitalizada com dor oncológica crônica, posto que valoriza os riscos, as incertezas, a ordem e a desordem que permeiam todo esse processo. ${ }^{6}$ Nessa conjuntura, sabe-se da importância do profissional de saúde valorizar a multidimensionalidade da dor oncológica crônica e gerenciar essa condição com base em estratégias que levem em consideração a idade, a cultura e a condição clínica da criança. ${ }^{5}$

Por essa razão, ressalta-se que compreender a complexidade que envolve a avaliação e o manejo da dor oncológica crônica revela-se necessário, uma vez que tais atividades não se baseiam apenas em elementos objetivos e técnicos, mas também englobam os aspectos interativos, subjetivos, o trabalho em equipe e todas as medidas necessárias para atenuar o sofrimento infantil e familiar. Sendo assim, tem-se como questão de pesquisa: como os profissionais de saúde avaliam e realizam o manejo da dor oncológica crônica da criança hospitalizada?

Com base no exposto, a presente pesquisa inova ao abordar os aspectos interativos e os significados que permeiam a avaliação e o manejo da dor oncológica crônica, sob o âmbito da complexidade do trabalho da equipe multiprofissional de saúde, visto que, quanto ao tema, a literatura tem focado predominantemente em questões técnicas da avaliação e do manejo profissional e/ou parental desse fenômeno., ${ }^{3,-10}$ Portanto, o objetivo deste estudo consiste em compreender a complexidade da avaliação e do manejo da dor oncológica crônica da criança hospitalizada. 
Avaliação e manejo da dor oncológica crônica em unidade de internação pediátrica | 4

\section{Método}

Estudo de abordagem qualitativa, desenvolvido com base no referencial metodológico da Grounded Theory $(\mathrm{GT})^{11}$ e no referencial teórico da Teoria da Complexidade na perspectiva de Edgar Morin. ${ }^{6}$

Os dados foram coletados pelo pesquisador principal por meio de entrevistas semiestruturadas gravadas em áudio e observação não participante, com 21 profissionais de saúde de uma Unidade de Internação Pediátrica (UIP) de um hospital público, no Rio de Janeiro, Brasil, especializado no tratamento de doenças hematológicas. A coleta de dados ocorreu entre os meses de agosto de 2014 e junho de 2015, nas dependências da UIP, tendo uma duração média de 20 minutos. A UIP está localizada no oitavo andar da instituição e conta com 13 leitos.

Os participantes do estudo foram definidos a partir do recurso da amostragem teórica cujo objetivo consiste em procurar pessoas e fatos que ajudem o pesquisador a tornar densas as categorias elaboradas. ${ }^{11} \mathrm{O}$ primeiro grupo amostral foi composto por sete enfermeiros, haja vista serem os profissionais responsáveis pelo gerenciamento do cuidado de enfermagem. As análises iniciais das entrevistas realizadas com os enfermeiros tornaram evidente a importância do trabalho em equipe, ressaltando a relação de interdependência entre os envolvidos no cuidado à criança. Em seguida, partindo dessa compreensão, sete técnicos de enfermagem foram entrevistados com o objetivo de compreender sua participação na avaliação e no gerenciamento da dor oncológica crônica, ao passo que, no âmbito do trabalho da equipe de enfermagem, são os profissionais envolvidos no cuidado direto à criança. Dada a relação de interdependência entre o trabalho da enfermagem e demais profissionais, foram entrevistados mais sete profissionais de saúde pertencentes a outras classes profissionais, a saber: duas médicas, duas fisioterapeutas, uma psicóloga, uma assistente social e uma farmacêutica. 
5 | Silva TP, Silva LJ, Silva IR, Ferreira MJC, Costa LS, Leite JL (In Memorian)

Todos os participantes foram recrutados por contato direto e se enquadraram nos critérios de inclusão: ter experiência mínima de um ano no cuidado à criança oncológica e esse mesmo período de vinculação profissional à instituição. Excluíram-se os profissionais de saúde que se encontravam em férias ou de licença no período da coleta de dados. Ressalta-se que o direcionamento para os demais grupos amostrais ocorreu mediante concordância do Comitê de Ética e Pesquisa (CEP).

As entrevistas com os participantes do primeiro grupo amostral tinham como questão norteadora: como você avalia e gerencia a dor oncológica crônica da criança hospitalizada? Para os participantes do segundo e terceiro grupos, as questões norteadoras foram: como você cuida da criança com dor oncológica crônica? Qual significado você atribui ao trabalho em equipe no cuidado à criança com dor oncológica crônica? Em função do recurso metodológico conhecido como amostragem teórica, as questões norteadoras elaboradas para o segundo e terceiro grupos da amostra emergiram a partir do pressuposto inicial da importância do trabalho em equipe e da relação de interdependência no trabalho interprofissional.

A observação não participante totalizou 54 horas, sendo realizada em cinco ocasiões durante o período diurno e empregada com a finalidade de compreender na prática os significados que orientam as atitudes, os comportamentos, as (re)ações e as interações estabelecidas entre os profissionais de saúde no cuidado à criança com dor oncológica crônica.

A análise do tipo comparativa foi realizada simultaneamente à coleta, sendo esta uma característica do método. A finalização da coleta de dados em cada grupo amostral foi determinada pelo recurso da saturação teórica. Os dados das entrevistas passaram inicialmente por uma codificação do tipo aberta, com análise linha a linha, gerando códigos preliminares que, agrupados por similaridades, deram origem aos códigos conceituais e estes comparados entre si geraram subcategorias e categorias. ${ }^{11}$ 
Avaliação e manejo da dor oncológica crônica em unidade de internação pediátrica | 6

Logo após, a codificação axial possibilitou relacionar as subcategorias entre si, determinando suas propriedades e dimensões. Por último, na codificação seletiva, a partir do refinamento dos conceitos que emergiram, definiu-se o fenômeno central do estudo. Em todas essas etapas de codificação também foram utilizados memorandos e diagramas como importantes ferramentas analíticas. ${ }^{11}$ Por fim, os resultados desta pesquisa passaram por uma validação, entre os meses de setembro e outubro de 2016, contando com a participação de cinco juízes, sendo três pesquisadores da área da Enfermagem com expertise na Grounded Theory e duas enfermeiras assistenciais do primeiro grupo amostral do presente estudo.

A pesquisa foi conduzida de acordo com os padrões éticos exigidos pelas Resoluções 466/2012 e 510/2016. Sendo assim, obteve aprovação do CEP da instituição onde o estudo foi desenvolvido, no dia 15/07/2015, sob parecer de número 355/14, CAAE 32795514.8.3001.5267, e do CEP da instituição proponente, no dia 03/10/2014, sob parecer de número 816.736, ambas tendo ocorrido em 2014. Todos os participantes assinaram o Termo de Consentimento Livre e Esclarecido. As falas dos enfermeiros estão identificadas pela letra $\mathrm{E}$, as dos técnicos de enfermagem pela letra $\mathrm{T}$, as das médicas pela letra $\mathrm{M}$, as da farmacêutica pelas letras $\mathrm{FC}$, as da psicóloga pela letra $\mathrm{P}$, as das fisioterapeutas pelas letras FS e as da assistente social pelas letras AS. Todas estão seguidas por um algarismo que se refere à ordem das entrevistas em cada grupo amostral (E1, T1, M1).

\section{Resultados}

O uso da Grounded Theory, como método de pesquisa qualitativa, possibilita a construção de categorias conceituais que quando relacionadas entre si são capazes de explicar o fenômeno de estudo. ${ }^{11}$ Dessa relação analítica, emergiu o fenômeno central "Gerenciamento do cuidado de enfermagem à criança hospitalizada com dor oncológica crônica: uma experiência de múltiplas inter-ações”. Este artigo apresenta a categoria: avaliação e manejo da dor oncológica crônica, composta por quatro subcategorias: necessitando trabalhar em equipe; 
7 | Silva TP, Silva LJ, Silva IR, Ferreira MJC, Costa LS, Leite JL (In Memorian)

interagindo com a criança e com seu familiar; significando a (re)avaliação da dor oncológica crônica; e descrevendo o manejo da dor oncológica crônica.

$\mathrm{Na}$ subcategoria necessitando trabalhar em equipe, entendeu-se que essa modalidade de trabalho é reconhecida pelos participantes como necessária, posto que a criança precisa ser atendida no conjunto de suas necessidades:

Eu acho fundamental o trabalho da equipe multidisciplinar porque a criança é vista de forma holística. (T6)

A criança com dor oncológica crônica precisa ser atendida por todos os profissionais [...] não existe só a enfermagem, o médico. É cada um na sua peculiaridade. (FC3)

Quando nós trabalhamos em equipe, as coisas fluem melhor. Na verdade, não dá para você tratar a saúde em partes separadas. (AS7)

O trabalho em equipe é extremamente importante para qualquer tipo de paciente e cada um tem sua função. Não adianta o médico querer resolver tudo sozinho que ele não vai conseguir avaliar, prescrever, reavaliar. (M1)

O trabalho em equipe possibilita ao enfermeiro agir com mais segurança, evitar danos, iatrogenias e gerenciar desordens:

Eu vou precisar falar com a equipe de fisioterapia para atuar melhor naquele membro afetado pela dor oncológica crônica [...] eu preciso do olhar do fisioterapeuta para manejar e perceber os limites de movimento daquele membro afetado pela dor. (E1)

Muitas vezes, eu tenho que conversar com outros profissionais, como o farmacêutico, para a gente conseguir acelerar o processo e resolver mais rápida a falta de medicação. (E2)

Quando a situação está muito complicada, eu procuro fazer reunião com o serviço social, com a CCIH [Comissão de Controle de Infecção Hospitalar] e com a chefia médica. Nós abordamos tratamento, acesso, direitos e deveres do acompanhante. (E5) 
Avaliação e manejo da dor oncológica crônica em unidade de internação pediátrica $\mid 8$

Nota-se que, para a avaliação e manejo da dor oncológica crônica, fazem-se necessárias múltiplas interações entre os profissionais de saúde, a criança e o familiar. A esse respeito, na subcategoria interagindo com a criança e com o seu familiar, revelou-se a importância do familiar no contexto hospitalar e a dificuldade de alguns profissionais em interagir com esse agente do cuidado:

A presença do familiar é fundamental, porque é o elo da criança. Se a mãe está ausente, a criança não fica à vontade com a gente. (E3)

Os pais são fundamentais. Nós acolhemos esses pais para que eles possam ter uma compreensão do diagnóstico para que eles com base nesse entendimento possam passar o significado para a criança. (P6)

As nossas dificuldades aqui, às vezes, são com os responsáveis porque eles acham que o medicamento e a fisioterapia vão zerar a dor da criança. (FS5)

Tem mãe que é totalmente fácil de lidar, agora têm mães que são difíceis. (T4)

Por outro lado, no âmbito do trabalho em equipe, os profissionais de saúde referiram apresentar uma relação afetiva e sem dificuldades interativas com a criança hospitalizada com dor oncológica crônica, fato observado durante observação não participante:

Eu consigo ter uma boa interação com a criança [...] eu consigo conversar, eu consigo perceber quando a criança não quer ser examinada, quando a criança está chateada com alguma coisa há toda uma psicologia que eu uso na interação. (M2)

A minha interação com a criança é ótima. (E6)

Aqui no hospital a gente cuida da criança com o máximo de carinho possível. (T5)

Eu não tenho muita dificuldade em interagir com a criança. (FS4) 
9 | Silva TP, Silva LJ, Silva IR, Ferreira MJC, Costa LS, Leite JL (In Memorian)

As interações com a criança e com o seu familiar favorecem a avaliação da dor oncológica crônica de forma mais efetiva. A esse respeito, na subcategoria significando a (re)avaliação da dor oncológica crônica, compreendeu-se que a avaliação e a reavaliação da dor são ações importantes para reduzir os seus impactos na qualidade de vida da criança:

É muito importante essa avaliação primária, como também a reavaliação, porque a criança oncológica sofre muito, tanto a família como os profissionais. (E6)

Você tem que partir do entendimento que a dor oncológica crônica é um incômodo para a criança e que ela vai atrapalhar a qualidade de vida da criança e o seu tratamento como um todo, por isso ela precisa ser avaliada sempre. (E7)

A avaliação da dor é muito importante porque é a partir dela que você observa a clínica da criança, a causa da dor. Você examina o paciente como um todo e a função é você intervir de imediato para acabar com o problema da criança aliviando seu sofrimento. (M2)

Uma avaliação abrangente da dor oncológica crônica inclui a identificação de sua origem e causa, ação realizada não só pelo enfermeiro, como também por outros profissionais de saúde. Nesse processo, os profissionais de saúde dispõem de instrumentos, os quais são utilizados para conhecer a intensidade da dor:

A gente avalia primeiro a causa da dor, se há alguma infecção associada. (M2)

Primeiro, nós levamos em consideração a causa da dor oncológica porque aqui nós temos aquelas crianças com uma dor crônica oncológica devido à metástase específica em uma determinada região. (FS5)

Para avaliar a dor, a gente se baseia nas escalas analógicas de cores, numérica e de faces. (E5) 
Avaliação e manejo da dor oncológica crônica em unidade de internação pediátrica | 10

No âmbito do trabalho em equipe, o relato da criança e do familiar é valorizado pela psicóloga na sua avaliação da dor. Isso significa que uma escuta qualificada é fundamental para acolher as demandas desse binômio:

A minha avaliação da dor, como psicóloga, implica em ouvir a criança porque no paciente falciforme existe a dor crônica que é física e é fácil de perceber, pois o paciente grita de dor. No paciente oncológico, depois que a criança entende todo o significado de sua doença, a dor crônica vai além. (P6)

O envolvimento do familiar na avaliação da dor oncológica crônica foi significado pelos profissionais de saúde como importante estratégia de ação. A observação não participante revelou que os profissionais de saúde apresentam uma escuta ativa ao relato da criança e do familiar sobre a experiência de dor, fato que reforça os depoimentos a seguir:

Eu vou muito nessas três bases: a face da criança, o relato da criança e o relato do acompanhante [...]. Eu considero muito importante o relato do acompanhante porque o acompanhante convive com essa criança e eles conhecem muito mais a criança que eu. (E2)

Eu acho importante porque quem mais lida com eles [crianças] são as mães e elas conhecem seus filhos, por isso, eu acho importante esse relato. (T1)

Nas crianças pequenas, a mãe é extremamente importante para identificar a dor. (M1)

A reavaliação da dor oncológica crônica foi significada como necessária, dada a sua importância em revelar a resposta da criança à terapêutica adotada:

Ela [reavaliação] é de total importância. Se você não reavaliar como é que você vai saber se a criança ainda tem dor? Por exemplo, você fez uma morfina, se você não reavaliar como é que você vai saber se a criança melhorou. (E2)

A reavaliação é para que a gente possa avaliar se aquele fármaco que está sendo utilizado regularmente está fazendo o efeito desejado e se a gente 
11 | Silva TP, Silva LJ, Silva IR, Ferreira MJC, Costa LS, Leite JL (In Memorian)

pode estar adicionando mais algum cuidado não só farmacológico, mas tentar intervir de alguma outra forma. (E1)

Alguns profissionais de enfermagem manifestaram dificuldades em (re)avaliar a dor, principalmente quando a criança está incapaz de verbalizá-la:

Às vezes, nem sempre a dor que a mãe diz que a criança está sentindo é a dor que realmente a criança está sentindo e você tem que saber diferenciar essas coisas. (E4)

Quando as crianças são maiores e conseguem verbalizar, a avaliação é mais fidedigna. Agora, quando não, fica muito complicada essa avaliação. (T3)

O processo de avaliação da dor implica no registro de suas características no prontuário da criança, favorecendo o acompanhamento de sua evolução clínica e o respaldo profissional:

O registro nos dá a base. É o nosso respaldo. É o que mostra a evolução do cuidado, se está havendo melhora ou não da criança. (E5)

O registro é importante porque como você vai ter respaldo se você não anotou?(T4)

A avaliação da dor oncológica crônica deve anteceder o seu manejo clínico. $\mathrm{Na}$ subcategoria descrevendo o manejo da dor oncológica crônica, constatou-se que o manejo farmacológico é importante e que os medicamentos são prescritos pelo médico, o qual segue a escada analgésica de dor:

A terapia farmacológica é muito importante para esse paciente, tem que existir porque o paciente não pode ficar com dor e tem que ser de uma forma racional. (FC3)

Se for uma dor mais fraca, nós usamos um analgésico menos potente. Se for uma dor mais intensa, nós utilizamos um opioide. (M1)

Se ele não está melhorando com uma analgesia básica, como a dipirona, nós passamos para uma analgesia mais forte, como é o tramal, até chegar na morfina. Nós vamos escalonando. (M2) 
Avaliação e manejo da dor oncológica crônica em unidade de internação pediátrica | 12

Nós começamos com o analgésico mais fraco até o mais forte. (T2)

A analgesia não farmacológica é também desenvolvida pelos profissionais de saúde. Eles a desenvolvem por meio do lúdico (brincadeira), da conversa, da massagem, do banho, da compressa morna ou fria e da promoção de conforto (posicionamento adequado, alongamento), também identificados durante a observação não participante:

Dependendo da situação da criança, eu vou adaptar a criança ao leito, eu vou proporcionar conforto, eu vou fazer massagem, eu vou dar um banho. (E1)

Eu uso o lúdico para tirar o foco do sofrimento porque quando a gente brinca, muitas vezes eles esquecem da dor [...] o lúdico é a minha maior estratégia aqui dentro. (E2)

Dependendo do estado em que a criança está, a medicação não surti muito efeito e você acaba tendo que partir para uma parte mais psicológica, tentar conversar com a criança, deixar o ambiente o mais calmo possível. (E4)

Dependendo da dor da criança, a gente faz bolsa de água quente ou gelo. (T6)

Eu trabalho o alongamento mais global, o posicionamento mais no leito e converso com a criança [...] tem dor que nós não conseguimos trabalhar fisicamente, é a base da conversa, do consolo, você fazendo uma atividade simples, um alongamento de membros inferiores ela já relata alivio [...] se a causa for física, neuromuscular, se a causa for na parte neuropática uma compressão, uma desnutrição nervosa que está levando aquela sensação de formigamento [...] nós trabalhamos com mobilização neural para trabalhar irrigação do nervo, nós vemos como está a hidratação da criança porque tudo isso ajuda, não é um enfoque único, há dor então vou usar calor ou frio.(FS5)

Com a criança nós brincamos, então realizamos o brincar [...] é também uma forma dela sair um pouco desse momento de tristeza que é o adoecer, 
13 | Silva TP, Silva LJ, Silva IR, Ferreira MJC, Costa LS, Leite JL (In Memorian)

ficar preso na internação e poder também extravasar um pouquinho, se alegrar, poder fantasiar, trazer o lúdico. (P6)

Ressalta-se que o uso da analgesia farmacológica e/ou não farmacológica exige dos profissionais de saúde conhecimento e habilidades para agir com segurança, favorecendo relações de confiança com seus pares, com a criança e com o familiar.

\section{Discussão}

Este estudo reforça que a avaliação e o manejo da dor oncológica crônica da criança hospitalizada demandam dos profissionais de saúde um trabalho em equipe visando ao atendimento das múltiplas necessidades da criança e da sua família. Assim, o relacionamento interprofissional é um elemento essencial para avaliação e manejo da dor, posto que torna possível um cuidado que contemple os componentes físico, espiritual, social e psicológico da dor oncológica crônica.

O trabalho em equipe evoca o princípio sistêmico ou organizacional da Teoria da Complexidade, no qual se entende que a organização de um todo comporta qualidades ou propriedades novas em relação as suas partes. Igualmente, o todo é menos que a soma de suas partes cujas qualidades são inibidas pela organização do conjunto, ${ }^{6}$ ou seja, o trabalho em equipe confere ao cuidado profissional novas propriedades quando realizado de forma efetiva, ao passo que possibilita a articulação de saberes disciplinares, favorecendo o cuidado integral.

A presença do familiar, muitas vezes representada pela figura materna, foi caracterizada pelos profissionais de saúde como fundamental, porém alguns manifestaram dificuldades em preservar esse relacionamento. A despeito desse fato, estudos revelam que é comum ocorrer conflitos entre os profissionais de saúde e o familiar da criança hospitalizada, visto que essa definição de papéis não está clara para ambos. ${ }^{12-13}$ Apesar disso, é importante o profissional de saúde valorizar a sua relação com o familiar da criança hospitalizada, haja vista esse último 
Avaliação e manejo da dor oncológica crônica em unidade de internação pediátrica $\mid 14$

desempenhar um papel de agente facilitador do processo de avaliação e manejo da dor, sobretudo nas situações em que a criança não consegue verbalizar esse fenômeno. ${ }^{14-15}$

Compreende-se que a família é uma unidade de complexidade humana, na qual se concentram os cuidados à criança. Trata-se de um concentrado biológico, psicológico, cultural e social muito forte, capaz de influenciar o comportamento e o desenvolvimento infantil. ${ }^{6}$

No que se refere à avaliação da dor, independente de sua causa, entende-se que se trata de um processo complexo e contínuo, sendo um pré-requisito para o seu correto manejo. ${ }^{16}$ Os participantes desta investigação avaliaram a dor oncológica crônica com base em três medidas: relato da criança, observação de sua expressão facial e comportamental e relato do familiar. A literatura destaca que o autorrelato, quando possível, é considerado a forma ideal para avaliar a dor na criança, uma vez que se configura como medida mais confiável da presença e severidade da dor. Todavia, ela também salienta que as escalas de autorrelato são consideravelmente complexas para as crianças em idade pré-escolar. ${ }^{17}$

No que tange ao envolvimento do familiar nas relações de cuidado, pesquisa realizada em Israel mostrou que $90 \%$ dos enfermeiros de uma unidade pediátrica referiram que é importante envolver a família na avaliação da dor da criança hospitalizada, porém, na prática, apenas $34 \%$ reportaram o envolvimento da mesma nesse processo. A maioria dos enfermeiros referiu avaliar a dor tendo como base a sua própria percepção $(89 \%)$, o autorrelato da criança $(86,5 \%)$ e o choro dela $(79 \%){ }^{18}$

Embora o relato do familiar seja importante na avaliação da dor, é preciso que o enfermeiro e os demais profissionais de saúde saibam ponderar sobre a real intensidade dessa condição na criança, ao passo que a literatura evidencia ocorrer divergências entre a percepção dos pais, da própria criança e dos profissionais de saúde. Sobre o exposto, estudo identificou que na comparação da avaliação da intensidade da dor feita por médicos, crianças e familiares, 
15 | Silva TP, Silva LJ, Silva IR, Ferreira MJC, Costa LS, Leite JL (In Memorian)

mediante o uso da escala numérica, a proporção de concordância entre as crianças e os médicos foi de $14,6 \%$, entre médicos e familiares $15 \%$ e entre crianças e familiares $40,1 \%{ }^{19}$

Por essa razão, é premente que os profissionais de saúde estejam devidamente qualificados e preparados para uma correta avaliação da dor. Isso envolve não só saber utilizar de forma efetiva os instrumentos disponíveis na literatura, como também educar os sentidos para uma percepção mais abrangente sobre a experiência de dor da criança, reconhecendo, contudo, a possibilidade de lidar com erros e ilusões. Na teoria da complexidade, compreende-se que a percepção é uma tradução e reconstrução cerebral dos estímulos ou sinais codificados pelos sentidos. Disso decorre a possibilidade de erros, incluindo os intelectuais, os quais são condicionados pela percepção. ${ }^{6}$

O processo de avaliação da dor oncológica crônica inclui o registro, no prontuário da criança, de suas características e das intervenções realizadas pelo profissional, de forma legível e completa. Contudo, estudos apontam a necessidade de avanços nos registros de dor e ressaltam as anotações profissionais como necessárias, pois ajudam na manutenção da segurança do paciente..$^{20-21}$

Com base nos resultados, é possível compreender que o manejo da dor oncológica crônica é feito pelo enfermeiro e demais profissionais de saúde por meio de analgesia farmacológica e não farmacológica. Quanto à analgesia farmacológica, ressalta-se a participação dos seguintes profissionais: médicos, enfermeiros, técnicos de enfermagem e farmacêuticos. A esse respeito, a literatura revela que a abordagem multidisciplinar baseada em terapia farmacológica e não farmacológica para o manejo da dor aguda e crônica resulta em efetiva melhora na qualidade de vida da população pediátrica com potencial redução de dependência aos opioides. ${ }^{22}$

Atualmente, recomenda-se que a analgesia farmacológica de crianças com câncer em tratamento de dor persistente seja baseada em duas etapas: para dor leve em crianças maiores de três meses, o paracetamol e o ibuprofeno se apresentam como medicamentos de primeira opção. Para analgesia de crianças com dor moderada a grave, opioides como morfina em doses baixas 
Avaliação e manejo da dor oncológica crônica em unidade de internação pediátrica | 16

devem ser considerados como primeira escolha. Para crianças menores de três meses, o paracetamol se apresenta como única opção. ${ }^{5}$

Nessa direção, um estudo constatou que os medicamentos não opioides foram mais utilizados (45\%) que os opioides (32\%) no manejo da dor de crianças com câncer avançado. ${ }^{2}$ Por outro lado, ressalta-se que os opioides são em maioria os medicamentos mais usados em crianças com dor oncológica crônica ou com dor persistente. ${ }^{5}$ Corroborando com o exposto, revisão integrativa identificou que os opioides são os medicamentos mais usados no manejo da dor oncológica, cabendo ao enfermeiro desenvolver cuidados na sua administração quanto à posologia, indicações, cumprimentos de horários e orientações à equipe de enfermagem. ${ }^{23}$

Dentre os opioides disponíveis, estão: morfina, tramadol, oxicodona, metadona, fentanil, entre outros. Sua disponibilidade nos países desenvolvidos é satisfatória, enquanto o seu acesso nos países em desenvolvimento é deficiente. Embora seja muito usado no manejo da dor oncológica, conforme evidenciado, estudo de revisão que objetivou avaliar a eficácia e os eventos adversos de opioides no manejo da dor oncológica em crianças e adolescentes de 0 a 17 anos identificou que não há evidências de ensaio clínico randomizado que apoiam ou refutam seu uso nessa faixa etária, haja vista que os testes se concentram em adultos. ${ }^{24}$

Em relação à analgesia não farmacológica, compreendeu-se que os profissionais de saúde do presente estudo desenvolvem massagem, banho, conversa, preparação do ambiente para proporcionar conforto, compressa morna ou fria, posicionamento no leito e atividades lúdicas. A esse respeito, um estudo de revisão integrativa identificou que entre as intervenções físicas e psicológicas utilizadas no manejo não farmacológico da dor oncológica no contexto pediátrico, estão aromaterapia, arte terapia, distração, hipnose, atividade física, posicionamento, massagem e a terapia cognitivo-comportamental multimodal. ${ }^{2}$

A utilização de estratégias de analgesia não farmacológica no manejo da dor oncológica crônica ressalta o seu aspecto multidimensional, complexo, fato que demanda dos profissionais 
de saúde saberes e práticas que transcendem a sua dimensão física. Nessa conjuntura, o conceito de dor total emerge na literatura como um princípio fundamental do cuidado paliativo, buscando melhorar a qualidade de vida de pacientes e familiares que enfrentam uma condição de saúde que ameace a continuidade da vida. ${ }^{25}$ Sendo assim, a dor total nos remete a pensar nos diferentes aspectos da vida afetados pela dor oncológica crônica, a saber: físico, psicológico, social e espiritual, perspectiva alinhada aos pressupostos da analgesia não farmacológica e da teoria da complexidade. ${ }^{26}$

Sobre o exposto, admite-se que considerar a analgesia não farmacológica como estratégia de ação possível para mitigar a dor oncológica crônica da criança hospitalizada significa romper com o paradigma da exclusão, segregação entre os diferentes saberes e disciplinas. Sob a ótica da complexidade, precisa-se da consciência de que a partir das ligações, cooperações e solidariedades entre os diversos saberes e práticas terapêuticas é possível reformar o pensamento em busca de um novo paradigma: o da complexidade, o qual se baseia na articulação, conexão entre as partes, o todo e seu o contexto. ${ }^{6}$

Este estudo apresenta como limitação o fato de apresentar apenas a perspectiva dos profissionais de saúde sobre o processo de avaliação e manejo da dor oncológica crônica da criança hospitalizada. Considerando que os resultados revelaram que os familiares e as crianças também estão envolvidos nessa atividade, admite-se a necessidade de estudos que investiguem a perspectiva desses participantes.

\section{Conclusão}

Os resultados deste estudo possibilitaram compreender que a avaliação e o manejo da dor oncológica crônica da criança hospitalizada são atividades complexas que demandam dos profissionais de saúde uma miríade de ações e interações, permeadas por aspectos objetivos e subjetivos. A avaliação da dor é realizada pelos profissionais de saúde com base no relato da criança, 
Avaliação e manejo da dor oncológica crônica em unidade de internação pediátrica $\mid 18$

do familiar e observação do comportamento da criança. Para tanto, estão disponíveis na instituição os seguintes instrumentos de avaliação: escala analógica de cores, escala numérica e escala de faces.

O manejo da dor é desenvolvido por meio da analgesia farmacológica e analgesia não farmacológica. A primeira segue a prescrição da equipe médica, a qual considera a escada analgésica, enquanto a segunda é realizada pelos demais profissionais de saúde. Nessa conjuntura, utilizam-se as seguintes estratégias não farmacológicas: o lúdico, a conversa, a massagem, o banho, a compressa morna ou fria e a promoção de conforto.

Enfatiza-se a importância do trabalho em equipe e do registro das informações sobre a avaliação e o manejo da dor no prontuário da criança, fato que permite acompanhar a evolução clínica da dor, além de conferir respaldo ao profissional sobre os cuidados desenvolvidos. A título de implicações para a prática de cuidados em saúde, os resultados mostraram a necessidade de os profissionais estabelecerem estratégias de interação e ação que favoreçam o trabalho em equipe e um bom relacionamento com a criança e com o familiar, além de apontar diretivas para avaliação e manejo da dor oncológica crônica. Salienta-se a necessidade de mais estudos sobre o tema a fim de aprofundar as discussões teóricas sobre o objeto de estudo.

\section{Referências}

1. Instituto Nacional de Câncer José Alencar Gomes da Silva (INCA). Incidência, mortalidade, morbidade hospitalar por câncer em crianças, adolescentes e adultos jovens no Brasil: informações dos registros de câncer e do sistema de mortalidade [Internet]. Rio de Janeiro (RJ): INCA; 2016 [acesso em 2020 jun 24]. Disponível em: https://www.inca.gov.br/publicacoes/livros/incidencia-mortalidade-emorbidade-hospitalar-por-cancer-em-criancas-adolescentes

2. Jibb LA, Nathan PC, Stevens BJ, Seto E, Cafazzo J, Stephens N, et al. Psychological and physical interventions for the management of cancer-related pain in pediatric and young adults patients: an integrative review. Oncol Nurs Forum. 2015;42(6):339-57. doi: https://doi.org/10.1188/15.ONF.E339-E357

3. Fortier MA, Wahi A, Bruce C, Maurer EL, Stevenson R. Pain management at home in children with cancer: a daily diary study. Pediatr Blood Cancer. 2014;61(6):1029-33. doi: https://doi.org/10.1002/pbc.24907 
4. Silva TP, Silva LJ, Ferreira MJC, Silva IR, Rodrigues BMRD, Leite JL. Aspectos contextuais sobre o gerenciamento do cuidado de enfermagem à criança hospitalizada com dor oncológica crônica. Texto Contexto Enferm. 2018;27(3):e3400017. doi: https://doi.org/10.1590/0104-070720180003400017

5. World Health Organization (WHO). WHO guidelines on the pharmacological treatment of persisting pain in children with medical illnesses. Geneva: WHO; 2012.

6. Morin E. O método 5: a humanidade da humanidade. 5ª ed. Porto Alegre (RS): Sulina; 2012.

7. Angelini P, Boydell KM, Breakey V, Kurkure PA, Muckaden MA, Bouffet E, et al. Pain management and use of opioids in pediatric oncology in India: a qualitative approach. J Glob Oncol. 2017;3(4):331-7. doi: https://doi.org/10.1200/JGO.2016.003483

8. Batalha LMC, Fernandes AM, Campos C, Gonçalves AMPMPC. Avaliação da dor em crianças com cancro: uma revisão sistemática. Referência [Internet]. 2015 [acesso em 2020 jun 24];4(5):119-27. Disponível em: http://www.scielo.mec.pt/scielo.php?script=sci_arttext\&pid=S087402832015000200014\&lng=en\&nrm=iso\&tlng=pt

9. Tutelman PR, Chambers CT, Stinson JN, Parker JA, Fernandez CV, Witteman HO, et al. Pain in children with cancer: prevalence, characteristics and parent management. Clin J Pain. 2018;34(3):198-206. doi: https://doi.org/10.1097/AJP.0000000000000531

10. Chung WW, Agbayani CG, Martinez A, Le V, Cortes H, Har K, et al. Improving children's cancer pain management in the home setting: development and formative evaluation of a web-based program for parents. Comput Biol Med. 2018;1(101):146-52. doi: https://doi.org/10.1016/j.compbiomed.2018.08.014

11. Strauss AL, Corbin J. Pesquisa qualitativa: técnicas e procedimentos para o desenvolvimento da teoria fundamentada. Porto Alegre: Artmed; 2008.

12. Silva TP, Silva IR, Leite JL. Interações no gerenciamento do cuidado de enfermagem a criança em condição crônica: revelando condições intervenientes. Texto Contexto Enferm. 2016;25(2):e1980015. doi: https://doi.org/10.1590/0104-07072016001980015

13. Silva TP, Silva MM, Valadares GV, Silva IR, Leite JL. Nursing care management for children hospitalized with chronic conditions. Rev Bras Enferm. 2015;68(4):556-63. doi: https://doi.org/10.1590/0034-7167.2015680410i

14. Aziznejadroshan P, Alhani F, Mohammadi E. Experiences of Iranian nurses on the facilitators of pain management in children: a qualitative study. Pain Res Treat. 2016;2016(6):1-7. doi: https://doi.org/10.1155/2016/3594240

15. Silva TP, Silva LJ, Rodrigues BMRD, Silva IR, Christoffel MM, Leite JL. Gerenciamento do cuidado a criança hospitalizada com dor oncológica crônica: condições intervenientes. Rev Bras Enferm. 2019;72(Suppl 1):190-7. doi: https://doi.org/10.1590/0034-7167-2017-0514 
Avaliação e manejo da dor oncológica crônica em unidade de internação pediátrica | 20

16. Cluxton C. The challenge of cancer pain assessment. Ulster Med J [Internet]. 2019 [cited 2020 Jun 24];88(1):43-6. Available from: https://www.ncbi.nlm.nih.gov/pmc/articles/PMC3992093/

17. Mahon P, Holsti L, Siden H, Stranhlendorf C, Turnham L, Giashi D. Using colors to assess pain in toddlers: validation of "the rainbow pain scale"- a proof-of-principle study. J Pediatr Oncol Nurs. 2015;32(1):40-6. doi: https://doi.org/10.1177/1043454214555197

18. Ziskk-Rony RY, Lev J, Haviv H. Nurses' report of in hospital pediatric pain assessment: examining challenges and perspectives. Pain Manag Nurs. 2015;16(2):112-20. doi: https://doi.org/10.1016/j.pmn.2014.05.003

19. Brudvick C, Moutte SD, Baste V, Morken T. A comparison of pain assessment by physicians, parents and children in an outpatient setting. Emerd Med J. 2017;34(3):138-44. doi: https://doi.org/10.1136/emermed-2016-205825

20. Song W, Eaton LH, Gordon DB, Hoyle C, Doorenbos AZ. Evaluation of evidence-based nursing pain management practice. Pain Manag Nurs. 2015;16(4):456-63. doi: https://doi.org/10.1016/j.pmn.2014.09.001

21. Muñoz-Alvaredo L, Vallecillo ML, Pérez JMJ, Martin-Gil B, Moreno MFM, Fernandez-Castro M. Prevalence, pain management and registration in Internal Medicine Units. Enferm Clín. 2018;s11308621(18):30266-3. doi: https://doi.org/10.1016/j.pmn.2018.11.004

22. Wren AA, Ross AC, D'Souza G, Almgren C, Feinstein A, Marshall A, et al. Multidisciplinary pain management for pediatric patients with acute and chronic pain: a foundational treatment approach when prescribing opioids. Children (Basel). 2019;6(2):33. doi: https://doi.org/10.3390/children6020033

23. Oliveira Junior NJ, Oliveira SBS, Migowski ER, Riegel F. Nurses' role in the non-pharmacological pain treatment in cancer pain. Rev Dor [Internet]. 2017 [cited 2020 Jun 24];18(3):261-5. Available from: https://www.scielo.br/pdf/rdor/v18n3/1806-0013-rdor-18-03-0261.pdf

24. Wiffen PJ, Cooper TE, Anderson AK, Gray AL, Grégoire MC, Ljungman G, et al. Opioids for cancerrelated pain in children and adolescents. Cochrane Database Syst Rev. 2017;19(7). doi: https://doi.org/10.1002/14651858.CD012564.pub2

25. Phenwan T. Relieving total pain in an adolescent: a case report. BMC Res Notes. 2018;11:265. doi: 10.1186/s13104-018-3368-8

26. Scarponi D, Pession A. Play theraphy to control pain and suffering in pediatric oncology. Front Pediatr. 2016;4:132. doi: https://doi.org/10.3389/fped.2016.00132

Editora Científica Chefe: Cristiane Cardoso de Paula Editora Associada: Nara Marilene Oliveira Girardon-Perlini 
Fomento / Agradecimento: Coordenação de Aperfeiçoamento de Pessoal de Nível Superior

\author{
Autor correspondente \\ Thiago Privado da Silva \\ E-mail: thiagopsilva87@gmail.com \\ Endereço: Avenida Aluizio da Silva Gomes, 50, Cavaleiros, Macaé, Rio de Janeiro. \\ CEP: 27930-560
}

\title{
Contribuições de Autoria
}

\section{1 - Thiago Privado da Silva}

Concepção ou desenho do estudo/pesquisa, análise e/ou interpretação dos dados, revisão final com participação crítica e intelectual no manuscrito.

\section{2 - Laura Johanson da Silva}

Concepção ou desenho do estudo/pesquisa, análise e/ou interpretação dos dados, revisão final com participação crítica e intelectual no manuscrito.

\section{3 - Italo Rodolfo Silva}

Revisão final com participação crítica e intelectual no manuscrito.

\section{4 - Maria José Carvalho Ferreira}

Revisão final com participação crítica e intelectual no manuscrito.

\section{5 - Luana dos Santos Costa}

Revisão final com participação crítica e intelectual no manuscrito.

\section{6 - Joséte Luzia Leite}

Revisão final com participação crítica e intelectual no manuscrito.

\section{Como citar este artigo}

Silva TP, Silva LJ, Silva IR, Ferreira MJC, Costa LS, Leite JL (In Memorian). Avaliação e manejo da dor oncológica crônica em unidade de internação pediátrica. Rev. Enferm. UFSM. 2021 [Acesso em: Anos Mês Dia]; vol.11 e31: 121. DOI: https://doi.org/10.5902/2179769247865 